\title{
Solving PDEs in irregular geometries with multiresolution methods I: Embedded Dirichlet boundary conditions 象 $^{2}$
}

\author{
Matthew G. Reuter ${ }^{\mathrm{a}, \mathrm{b}}$, Judith C. Hill ${ }^{\mathrm{a}}$, Robert J. Harrison ${ }^{\mathrm{a}, \mathrm{c}, *}$ \\ a Computer Science and Mathematics Division, Oak Ridge National Laboratory, Oak Ridge, TN 37831-6367, USA \\ b Department of Chemistry, Northwestern University, Evanston, IL 60208-3113, USA \\ c Department of Chemistry, University of Tennessee, Knoxville, TN 37996, USA
}

\section{A R T I C L E I N F O}

\section{Article history:}

Received 2 September 2010

Received in revised form 1 July 2011

Accepted 2 July 2011

Available online 10 August 2011

\section{Keywords:}

Multiresolution analysis

Domain embedding techniques

Electrostatics

\begin{abstract}
A B S T R A C T
In this work, we develop and analyze a formalism for solving boundary value problems in arbitrarilyshaped domains using the MADNESS (multiresolution adaptive numerical environment for scientific simulation) package for adaptive computation with multiresolution algorithms. We begin by implementing a previously-reported diffuse domain approximation for embedding the domain of interest into a larger domain (Li et al., 2009 [1]). Numerical and analytical tests both demonstrate that this approximation yields non-physical solutions with zero first and second derivatives at the boundary. This excessive smoothness leads to large numerical cancellation and confounds the dynamically-adaptive, multiresolution algorithms inside MADNESS. We thus generalize the diffuse domain approximation, producing a formalism that demonstrates first-order convergence in both near- and far-field errors. We finally apply our formalism to an electrostatics problem from nanoscience with characteristic length scales ranging from 0.0001 to $300 \mathrm{~nm}$.
\end{abstract}

(c) 2011 Elsevier B.V. All rights reserved.

\section{Introduction}

Boundary value problems (BVPs) in irregular domains arise in many applications, ranging from fluid dynamics to photonics, and present additional computational challenges compared to their analogues in regular domains. First is the issue of function representation. Uniform, structured grids are often inefficient, requiring smaller-than-desirable grid spacings to resolve irregularities in the domain's shape. Unstructured meshes can adequately handle any irregularities; however, their generation is both expensive and problem-specific. Second, the imposition of boundary conditions can be non-trivial, depending on the choice of function representation. Multiresolution methods ameliorate many of the first concerns by providing efficient, adaptive representation schemes that hone in on computationally-troublesome regions to effectively utilize the available computational resources. Unfortunately, multiresolution methods perform best on regular domains; enforcing boundary conditions on an arbitrary surface is not straightforward.

\footnotetext{
This manuscript has been authored by a contractor of the U.S. Government under Contract No. DE-AC05-000R22725. Accordingly, the U.S. Government retains a non-exclusive, royalty-free license to publish or reproduce the published form of this contribution, or allow others to do so, for U.S. Government purposes.

* Corresponding author at: Computer Science and Mathematics Division, Oak Ridge National Laboratory, Oak Ridge, TN 37831-6367, USA.

E-mail address: harrisonrj@ornl.gov (R.J. Harrison).
}

Domain embedding techniques seem ideal for using multiresolution methods on irregular domains; as the name suggests, they embed the irregular domain into a larger, and usually simpler, domain. The two main obstacles to domain embedding techniques are (i) extending all pertinent quantities into the larger domain, and (ii) mapping the original surface BVP into the larger domain. The diffuse domain approximation (DDA) confronts these issues by broadening the embedded boundary into a finite-width boundary layer and using this finite-width layer to smoothly switch from the desired PDE in the interior to a simple PDE in the exterior [1]. Auxiliary source terms are introduced near the boundary layer to enforce the desired boundary condition, and a variety of methods can be employed to solve the resulting volume problem in the larger domain. Li et al. [1] derived several DDAs by matching the interior and exterior solutions at the boundary; however, no analytical or quantitative numerical investigations were reported to assess solution quality on the boundary or in the far-field regions (interior or exterior).

Our primary goals in this communication are threefold. First, we aim to quantitatively explore the efficacy of DDAs for producing acceptable solutions on the boundary and in the domain of interest. Second, we seek to combine the DDA with multiresolution methods, enabling general and practical techniques for solving BVPs in irregular domains. Third, we want to extend the MADNESS (multiresolution adaptive numerical environment for scientific simulation) environment [2,3], previously reported by us, to handle embedded boundary conditions. MADNESS's initial 
applications in chemistry, atomic and molecular physics, nuclear physics, solid-state physics, and fluids [3-7] have so far only required free-space or periodic boundary conditions on regular domains; the ability to treat embedded problems will expand MADNESS's utility to new applications, including solvation models and electromagnetism. In this paper, we analyze and improve upon previously-reported DDAs [1,8] for embedding irregular domains inside larger domains and then demonstrate them, using MADNESS, with an example from nanoscience.

We commence by presenting pertinent details of the multiresolution refinement algorithms employed by MADNESS in Section 2. Section 3 introduces the DDA used in this work, which is inspired by the previously-reported DDAs [1]. We proceed, in Section 4, to implement this DDA in MADNESS and numerically assess its robustness with several test problems. While the DDA properly converges to the correct solution in some cases, other examples exhibit slow and/or stalled convergence. The analytical results presented in Appendix A expose the origins of these failures and accurately determine the viability of the DDA. Finally, we apply our DDA to an electrostatics problem relevant to nanoscience in Section 5, calculating the electrostatic potential affected by nanometer-scale structures.

\section{Multiresolution analysis in MADNESS}

MADNESS is a high-level environment for solving integral and differential equations in many dimensions. Applications are composed in terms of functions and integral/differential operators, and computation appears to the user as being basis-set free. To preserve this high-level composition and to maintain the underlying guarantees of precision, we seek methods to incorporate boundary conditions that are compatible with the existing "analysis-based" approach. In particular, we wish to avoid incorporating either special elements or meshes not controlled by the multiresolution algorithms, since these will make it hard to maintain the expected guarantees of precision and will destroy the illusion of basis-free computation.

Multiresolution analysis in multiwavelet bases [9] enables construction of fast algorithms with guaranteed precision (e.g., for the multiplication of two functions) as well as fast application of integral operators (e.g., the Coulomb and bound-state Helmholtz Green's functions) $[3,4,9]$. The guarantee of precision is provided by dynamic adaptive refinement of the mesh underlying the function resulting from application of an operator to one or more input functions.

The underlying discontinuous spectral-element basis is comprised of the tensor product of Legendre polynomials within each adaptively-refined element. By construction, this basis facilitates sparse representations of functions and many physically-important integral operators, including convolution with the Coulomb Green's function $[3,4,9]$. For many problems, the integral form has superior numerical properties to the corresponding differential form, and, hence, an important feature of MADNESS is the ability to apply many integral operators using a single block-sparse, matrix-vector product. Furthermore, this basis quantifies both local error and local smoothness, which, in turn, enables adaptive, local refinement (refinement only occurs if the local error in the approximation is above a specified truncation threshold). Finally, the local error allows estimation of the global error, leading to precision guarantees for both representing a function and applying an operator to a function. More details on this basis are presented in Ref. [9] (Section 3.3.2 therein is particularly relevant).

\section{Diffuse domain approximation}

Li et al. presented DDAs for numerous differential operators subject to either Dirichlet-, Neumann-, or Robin-type boundary conditions [1]. For simplicity, this paper will focus on the problem

$\nabla^{2} u(\mathbf{x})=f(\mathbf{x}), \quad \mathbf{x} \in \Omega_{D}$,

$u(\mathbf{x})=d(\mathbf{x}), \quad \mathbf{x} \in \partial \Omega_{D}$

for an irregular domain $\Omega_{D}$ with boundary $\partial \Omega_{D}$. Extensions to other differential operators and/or boundary conditions are straightforward.

Embedding $\Omega_{D}$ into a larger domain $(\Omega)$ with a DDA requires a domain mask $\left[\varphi_{\varepsilon}(\mathbf{x})\right]$ that is 1 inside $\Omega_{D}$, is 0 outside $\Omega_{D}$, and smoothly varies from 0 to 1 in a layer of width $\mathcal{O}(\varepsilon)$ around $\partial \Omega_{D}$ $\left(\varepsilon \rightarrow 0^{+}\right)$. The DDA we study here is

$\nabla^{2} u_{\varepsilon}(\mathbf{x})-\alpha(\varepsilon) S_{\varepsilon}(\mathbf{x})\left[u_{\varepsilon}(\mathbf{x})-d(\mathbf{x})\right]=\varphi_{\varepsilon}(\mathbf{x}) f(\mathbf{x}), \quad \mathbf{x} \in \Omega$,

where $u_{\varepsilon}$ approximates the exact solution $u\left(\lim _{\varepsilon \rightarrow 0^{+}} u_{\varepsilon}=u\right)$, $\alpha(\varepsilon)$ is a penalty for enforcing the boundary condition, and $S_{\varepsilon}$ approximates the surface delta function on $\partial \Omega_{D}$ as $\varepsilon \rightarrow 0^{+}$. We extend $f$ and $d$ to be constant along surface normals in the exterior domain.

This DDA deviates from the previous work in two key ways. First, Eq. (2) generalizes a DDA from Ref. [1]; only $\alpha(\varepsilon)=\varepsilon^{-2}$ was considered there. As will be seen, vide infra, MADNESS's multiresolution methods inadequately resolve the boundary layer when $\alpha(\varepsilon)=\varepsilon^{-2}$, resulting in poor solution quality (and thus prompting us to generalize the DDA). Second, we choose $\varphi_{\varepsilon}$ and $S_{\varepsilon}$ differently to facilitate an analytical analysis of these problems when combining MADNESS with DDAs. In this work we employ

$S_{\varepsilon}(\mathbf{x})=\frac{1}{\varepsilon \sqrt{2 \pi}} \exp \left(-\frac{s(\mathbf{x})^{2}}{2 \varepsilon^{2}}\right)$

and

$\varphi_{\varepsilon}(\mathbf{x})=\frac{1}{2}\left[1-\operatorname{erf}\left(\frac{s(\mathbf{x})}{\varepsilon \sqrt{2}}\right)\right]$,

where $s$ gives the signed, minimum distance from the surface; $s(\mathbf{x})<0$ in the interior domain and $s(\mathbf{x})>0$ in the exterior. In the results that follow, all reported trends are duplicated if $\varphi_{\varepsilon}$ and $S_{\varepsilon}$ from Ref. [1] are used instead.

Since MADNESS works most efficiently with integral operators, we use the Coulomb operator's free-space Green's function, $G\left(\mathbf{x}, \mathbf{x}^{\prime}\right)=-\left(4 \pi\left|\mathbf{x}-\mathbf{x}^{\prime}\right|\right)^{-1}$ in three dimensions, to invert the Laplacian in Eq. (2). This produces

$$
\begin{aligned}
& u_{\varepsilon}(\mathbf{x})+\alpha(\varepsilon) \int_{\Omega} \mathrm{d}^{3} \mathbf{x}^{\prime} G\left(\mathbf{x}, \mathbf{x}^{\prime}\right) S_{\varepsilon}\left(\mathbf{x}^{\prime}\right) u_{\varepsilon}\left(\mathbf{x}^{\prime}\right) \\
& =\int_{\Omega} \mathrm{d}^{3} \mathbf{x}^{\prime} G\left(\mathbf{x}, \mathbf{x}^{\prime}\right)\left[\alpha(\varepsilon) S_{\varepsilon}\left(\mathbf{x}^{\prime}\right) d\left(\mathbf{x}^{\prime}\right)-\varphi_{\varepsilon}\left(\mathbf{x}^{\prime}\right) f\left(\mathbf{x}^{\prime}\right)\right],
\end{aligned}
$$

which is solved using the generalized minimal residual (GMRES) method [10].

Before proceeding, we note that the exact Green's function (as opposed to the free-space Green's function) would obviate the need for linear solvers. This exact Green's function would be problem-specific-depending on both the shape of the domain and the desired boundary condition-making it difficult to obtain and, most likely, inefficient to use. The image method is an alternative method for using only the free-space Green's function; however, its utility is limited to particular differential operators on sufficiently regular domains [11]. Our method is not hampered by either of these restrictions. 
Table 1

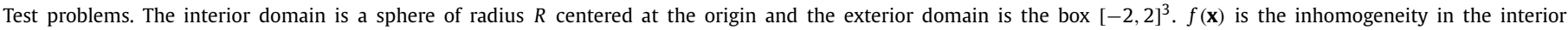

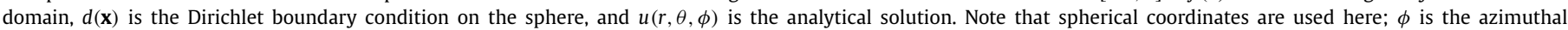
angle and is not related to the domain mask function $\left(\varphi_{\varepsilon}\right)$.

\begin{tabular}{|c|c|c|c|c|}
\hline Problem & $f(\mathbf{x})$ & $d(r=R, \theta, \phi)$ & $u(r \leqslant R, \theta, \phi)$ & $u(r>R, \theta, \phi)$ \\
\hline 1 & 0 & 1 & 1 & $(r / R)^{-1}$ \\
\hline 2 & 0 & $\cos (\theta)$ & $(r / R) \cos (\theta)$ & $(r / R)^{-2} \cos (\theta)$ \\
\hline 3 & 0 & $\left(3 \cos ^{2}(\theta)-1\right) / 2$ & $(r / R)^{2}\left(3 \cos ^{2}(\theta)-1\right) / 2$ & $(r / R)^{-3}\left(3 \cos ^{2}(\theta)-1\right) / 2$ \\
\hline 4 & $6 R^{-2}$ & 1 & $(r / R)^{2}$ & $(r / R)^{-1}$ \\
\hline
\end{tabular}
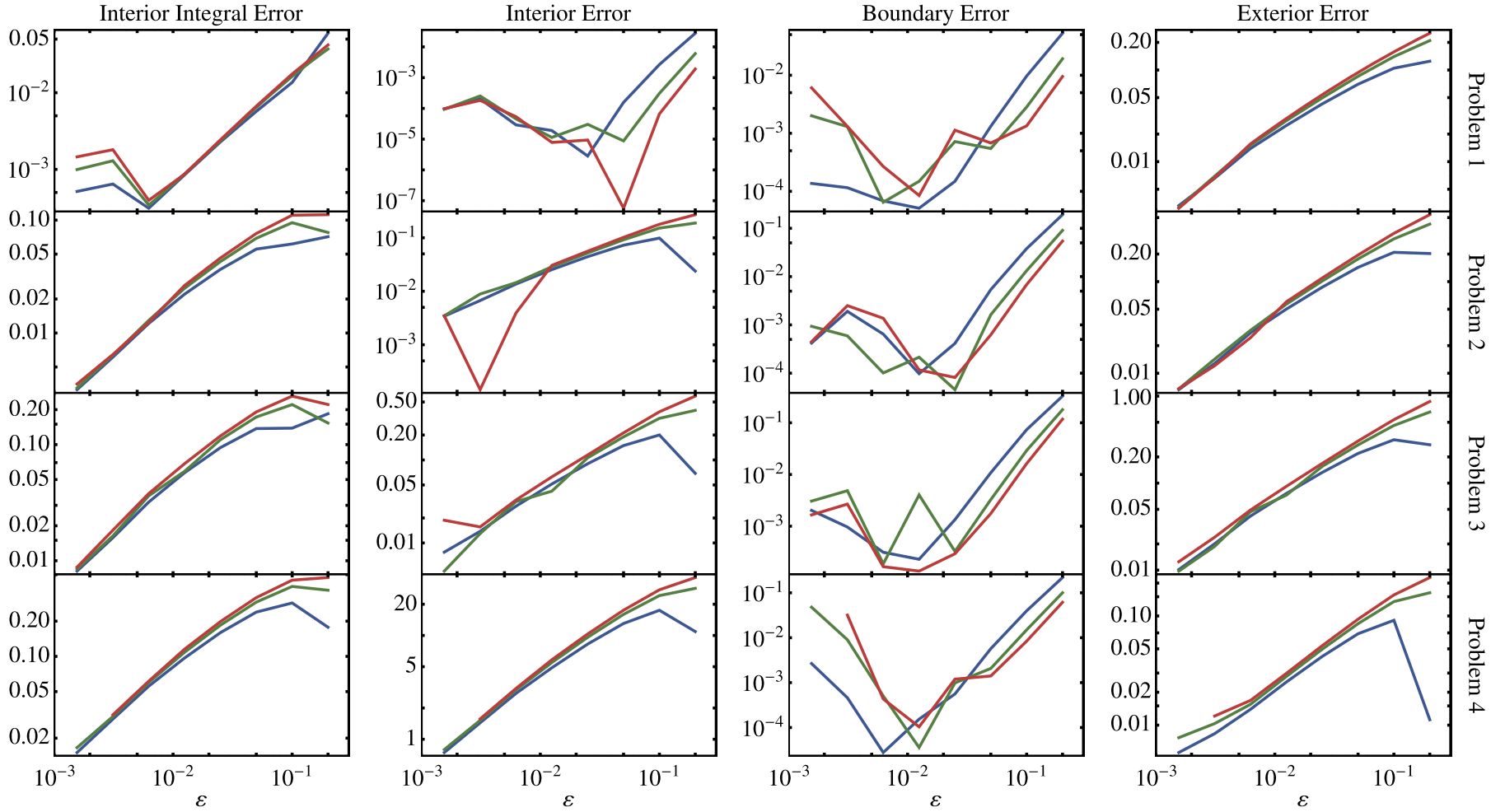

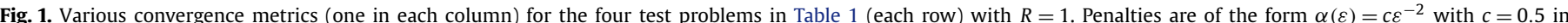

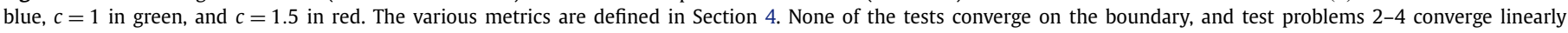

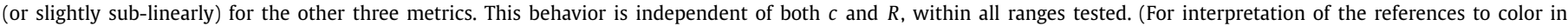
this figure legend, the reader is referred to the web version of this article.)

\section{Test problems \& numerical results}

Four test problems will be used to evaluate the efficacy of this DDA [Eqs. (2) and (5)] inside MADNESS. Each problem considers $\Omega_{D}$ to be the interior of a sphere of radius $R<2$ centered at the origin and $\Omega$ to be the box $[-2,2]^{3}$. Table 1 lists these test problems, along with their analytical solutions. Furthermore, four criteria will be used to assess the convergence of $u_{\varepsilon} \rightarrow u$ : (i) the interior integral error,

$\left\|\varphi_{\varepsilon}\left(u-u_{\varepsilon}\right)\right\|_{2} \equiv\left[\int_{\Omega} \mathrm{d}^{3} \mathbf{x} \varphi_{\varepsilon}(\mathbf{x})^{2}\left(u(\mathbf{x})-u_{\varepsilon}(\mathbf{x})\right)^{2}\right]^{1 / 2} ;$

and the relative error at specific points (ii) in the interior domain $\left(\Omega_{D}\right)$, (iii) on the boundary $\left(\partial \Omega_{D}\right)$, and (iv) in the exterior domain $\left(\Omega \backslash \Omega_{D}\right)$. We use $(0,0, R / 10)$ for the interior error, $(0,0, R)$ for the boundary error, and $(0,0,2)$ for the exterior error. Note that the exterior error is generally an irrelevant metric; however, since test problems 1-3 are homogeneous, it assesses any differences between convex and concave boundaries.

Using the penalty from Ref. [1] as a starting point, Fig. 1 displays the four convergence criteria for the four test problems when $R=1$ and $\alpha(\varepsilon) \propto \varepsilon^{-2}$ (the green lines show the previouslyreported DDA, $\alpha(\varepsilon)=\varepsilon^{-2}$ ). Regardless of the penalty's proportion- ality constant, only the exterior error appears to converge for test problem 1, and it does so linearly. The other test problems exhibit linear (or slightly sublinear) convergence in the interior integral, interior, and exterior metrics; however, they still fail to converge on the boundary. Convergence at the boundary is superlinear, usually better than cubic, when $\varepsilon \gtrsim 0.025$, but is stymied as $\varepsilon$ becomes smaller.

These convergence difficulties were not reported, and probably not observed, by Li et al. [1] for two reasons. First, the problems are not manifest unless $\varepsilon \lesssim 0.01$, and the previous work focused on $\varepsilon \geqslant 0.025$. Second, as discussed in Appendix A, $\alpha(\varepsilon) \propto \varepsilon^{-2}$ fails to trigger MADNESS's adaptive refinement algorithms at the boundary layer. Inadequate resolution of the boundary layer results in poor solution quality and arrested convergence. The analysis in Appendix A further reveals that $\alpha(\varepsilon) \propto \varepsilon^{-2}$ (i) over-penalizes at the boundary (at the expense of the interior and exterior regions) and (ii) causes an undesirable loss of precision as $\varepsilon \rightarrow 0^{+}$.

Reducing the penalty to $\alpha(\varepsilon) \propto \varepsilon^{-1}$ appears to remedy the convergence maladies, as seen in Fig. 2 and as discussed in Appendix A. First, all four convergence criteria converge linearly for all four test problems. The kinks in the interior and exterior errors are caused by transitions from over-estimating to under-estimating the true solution at the point of interest (or vice versa); averaging over the domain (the interior integral error) removes these effects. 

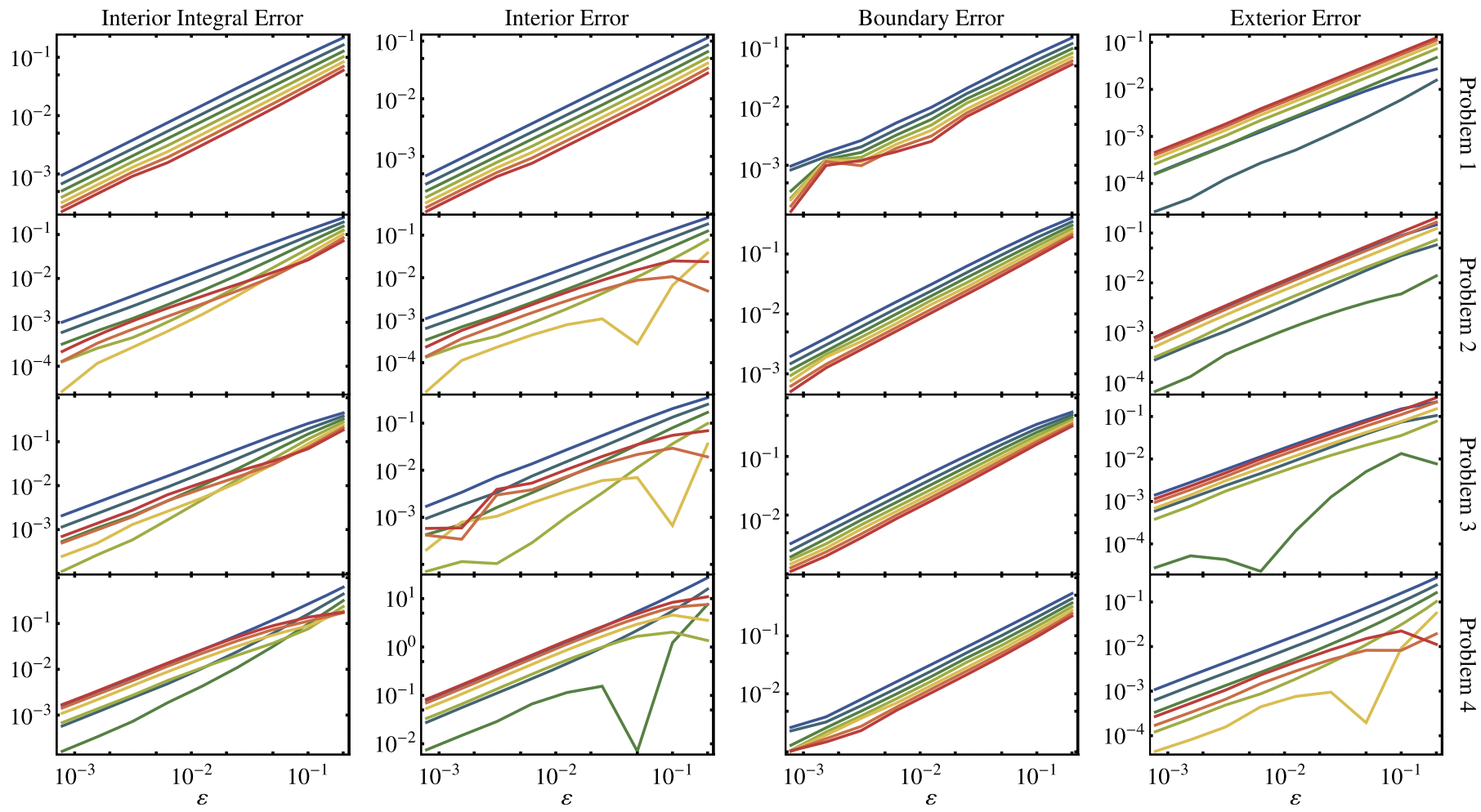

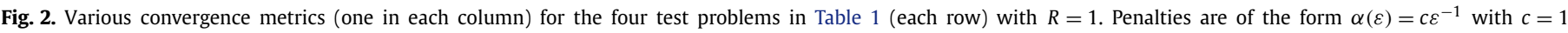

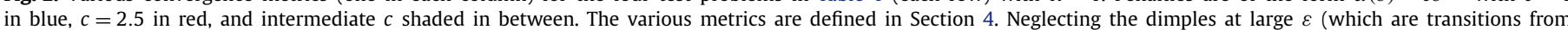

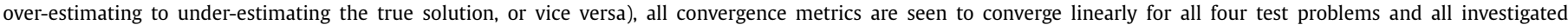

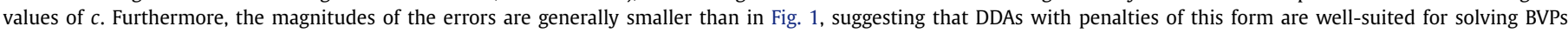
with Dirichlet-type boundary conditions. (For interpretation of the references to color in this figure legend, the reader is referred to the web version of this article.)

Second, the errors reported in Fig. 2 are generally smaller than those in Fig. 1, particularly for metrics other than the boundary error. The boundary errors when $\alpha(\varepsilon) \propto \varepsilon^{-1}$ converge slower and are larger than when $\alpha(\varepsilon) \propto \varepsilon^{-2}$ with $\varepsilon \gtrsim 0.025$; however, convergence when $\alpha(\varepsilon) \propto \varepsilon^{-1}$ is not limited to large $\varepsilon$. Taken collectively, the global convergence and improved solution quality throughout the interior domain suggest that penalties of the form $\alpha(\varepsilon) \propto \varepsilon^{-1}$ produce an acceptable DDA for solving BVPs with Dirichlet-type boundary conditions.

\subsection{Computational costs}

The computational cost of our method depends on the size of $\varepsilon$ relative to the size of the total computational domain $(\Omega)$. Smaller values of $\varepsilon$ require additional refinement, which in turn increases the computational cost. Our code was initially written and tested on a 2009 MacBook Pro with 4 GB RAM; the test problems with large $\varepsilon(\gtrsim 0.1)$ easily ran on this machine. Test problems with smaller $\varepsilon$, which yielded a sharper interface description, were run on Jaguar at the National Center for Computational Sciences because of the increased memory costs for the additional refinement required. The example nanoscience problem in Section 5 was also run on Jaguar, using 14,400 cores for approximately 10 minutes. No effort was made to optimize the code.

\section{Example: Electrostatics in nanoscale systems}

Nanometer-sized (nanoscale) systems often exhibit intriguing behavior compared to larger-sized systems, offering advances in applications such as sensors, solar cells, and catalysts. These systems are difficult to computationally model due to their numerous length scales, not to mention the various physical processes occurring on these disparate scales.

For example, the scanning tunneling microscope (STM) is a powerful device for investigating surface chemistry that brings a sharp metallic tip into the vicinity of a surface and measures electric current through the junction [12]. The application of a bias between the tip and surface is vital for the STM's operation, and illumination of the junction by a laser can also be desirable. Thus, the relevant length scales are (i) the dimensions of the STM tip-one or several atoms in diameter at the apex $[\mathcal{O}(0.5 \mathrm{~nm})]$ and $\mathcal{O}(>300 \mathrm{~nm})$ in length; (ii) the dimensions of the surface, $\mathcal{O}(>300 \mathrm{~nm})$; (iii) the distance between the tip and the surface, $\mathcal{O}(5 \mathrm{~nm})$; and (iv) the electronic $[\mathcal{O}(0.01-0.1 \mathrm{~nm})]$ and nuclear $[\mathcal{O}(0.0001 \mathrm{~nm})]$ charge densities from molecules in the gap. Modeling a tip alone is a challenging computational task because of its disparate dimensions [13], highlighting the advantages of using MADNESS for simulating these systems.

An electrostatics framework can be used to describe the junction between a STM and a surface in the absence of laser irradiation, such that

$\nabla^{2} V(\mathbf{x})=-\rho(\mathbf{x}) / \varepsilon_{0}$,

where $V$ is the electrostatic potential, $\rho$ is the external charge density, and $\varepsilon_{0}$ is the permittivity of free space (assuming our domain is in a vacuum). The applied bias enters through Dirichlettype boundary conditions; $V(\mathbf{x})$ is specified on the boundaries of the tip and of the surface. The sample system we consider is as follows. The STM tip is modeled by a paraboloid, has an electrostatic potential of $0.5 \mathrm{~V}$, and is placed $10 \mathrm{~nm}$ above a surface at $-0.5 \mathrm{~V}$. A hydrogen molecule is placed in the center of the 

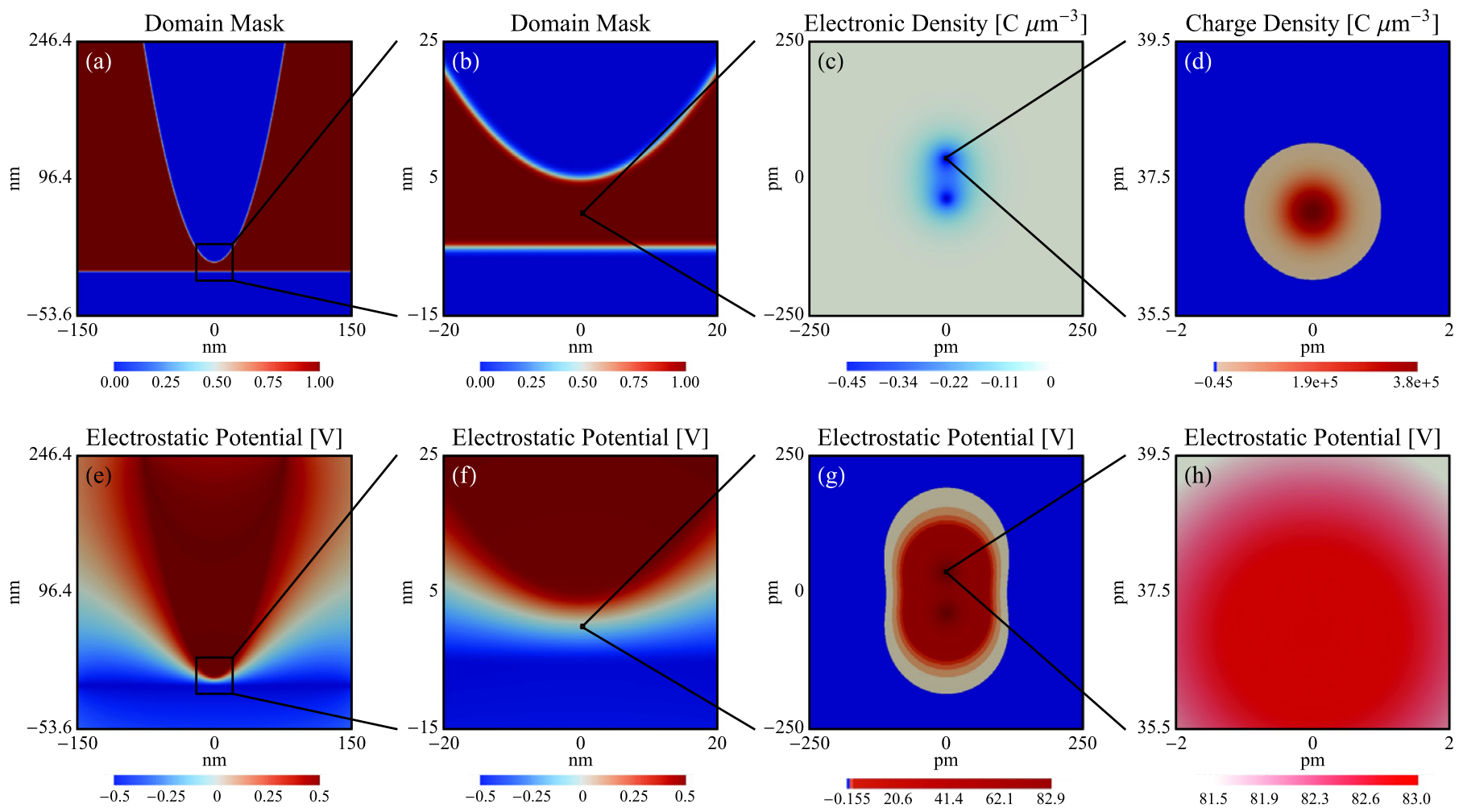

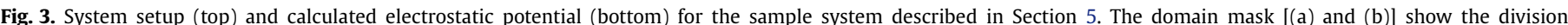

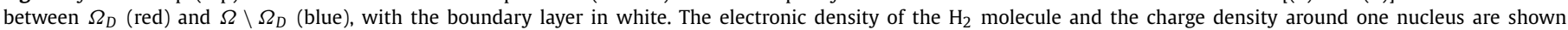

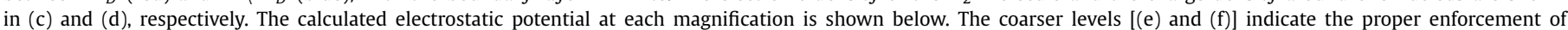

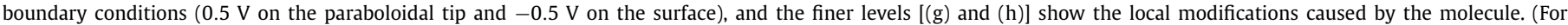
interpretation of the references to color in this figure legend, the reader is referred to the web version of this article.)

gap, contributing an electronic density. ${ }^{1}$ The nuclear charge densities are approximated by sharply-peaked Gaussians. For use in the DDA, $\alpha(\varepsilon)=1.75 / \varepsilon$ and $\varepsilon=0.6 \mathrm{~nm}$. Finally, Fig. 3 shows both the system setup and the electrostatic potential calculated with Eq. (5) in MADNESS; the seven orders of length scales are not problematic.

The electrostatic potential in such a STM junction provides the electric field,

$\vec{E}(\mathbf{x})=-\vec{\nabla} V(\mathbf{x})$,

which is expected to be large since the distance between the STM tip and the surface is small. This electric field can induce molecular excitation and/or motion, leading to interesting physics, including tip-enhanced Raman spectroscopy [15] and coherent control [16]. Finally, extending this work to time-dependent problems adds layers of computational complexity (see Refs. [13,17] for discussions of modeling electrodynamics in nanoscale systems); such studies will be reported at a later time.

\section{Conclusions}

In this communication, we have demonstrated that embedded domain techniques and diffuse domain approximations, in particular, are capable of enforcing Dirichlet boundary conditions on arbitrarily-shaped domains when using adaptive multiresolution methods. Specifically, we have shown that the penalization term in the DDA greatly affects the convergence behavior of the solution both inside the domain and on the boundary (i.e., enforce-

\footnotetext{
1 For simplicity, the hydrogen molecule's electronic density was obtained from an independent quantum mechanical calculation using the GAMESS package [14]. The density is expressed as a sum of Gaussian basis functions.
}

ment of the prescribed boundary condition). The penalization term $\alpha(\varepsilon) \propto \varepsilon^{-1}$ yields linear error convergence as $\varepsilon \rightarrow 0^{+}$for analytical test problems. We have further demonstrated the robustness of this methodology for a realistic nanoscience problem with multiple length scales of interest.

Though not explicitly explored, this approach can be easily extended to treat both Neumann- and Robin-type boundary conditions as well as moving boundaries in transient problems [1]. Demonstration of these capabilities will be forthcoming in a future communication.

\section{Acknowledgements}

We are grateful to Diego A. Galindo, George I. Fann, W. Scott Thornton, and William A. Shelton Jr. for interesting conversations. M.G.R. thanks the DoE Computational Science Graduate Fellowship Program (Grant No. DE-FG02-97ER25308) for a graduate fellowship and the opportunity to conduct this research. This research is sponsored by the Office of Advanced Scientific Computing Research; U.S. Department of Energy. The work was performed at the Oak Ridge National Laboratory, which is managed by UT-Battelle, LLC under Contract No. De-AC05-000R22725 and employed resources of the National Center for Computational Sciences at Oak Ridge National Laboratory.

\section{Appendix A. Analytical analysis}

Figs. 1 and 2 show how the form of the penalization term in the studied DDA has a large effect on the DDA's efficacy. Most notably, $\alpha(\varepsilon) \propto \varepsilon^{-2}$ has trouble converging on the boundary for small $\varepsilon$, whereas $\alpha(\varepsilon) \propto \varepsilon^{-1}$ appears to exhibit global, first-order convergence. This appendix offers an analytical/asymptotic investigation into this behavior. 
Table 2

Expansion coefficients of the Taylor series about $r=R=1$ [Eq. (A.1)] for solutions of test problem 2 [Eq. (2) and Table 1].

\begin{tabular}{lll}
\hline Coefficient & $\alpha(\varepsilon)=c \varepsilon^{-2}$ & $\alpha(\varepsilon)=c \varepsilon^{-1}$ \\
\hline$u_{0}$ & $1-2 \sqrt{2 \pi} c^{-1} \varepsilon^{3}+\mathcal{O}\left(\varepsilon^{4}\right)$ & $1-48 \pi c^{-1}\left(16 \pi+3 c \sqrt{2 \pi}+c^{2}\right)^{-1} \varepsilon+\mathcal{O}\left(\varepsilon^{2}\right)$ \\
$u_{1}$ & $-3 \pi c^{-2} \varepsilon^{2}+\mathcal{O}\left(\varepsilon^{3}\right)$ & $-3 \pi\left(6 \pi+3 \sqrt{2 \pi} c+c^{2}\right)^{-1}+\mathcal{O}(\varepsilon)$ \\
$u_{2}$ & $-(12+c) \sqrt{2 \pi} c^{-2} \varepsilon+\mathcal{O}\left(\varepsilon^{2}\right)$ & $-12 \sqrt{2 \pi}\left(16 \pi+3 \sqrt{2 \pi} c+c^{2}\right)^{-1} \varepsilon^{-1}+\mathcal{O}\left(\varepsilon^{0}\right)$ \\
$u_{3}$ & $-\sqrt{2 \pi}(4 c \varepsilon)^{-1}+\mathcal{O}\left(\varepsilon^{0}\right)$ & $-\sqrt{2 \pi} c\left(6 \pi+3 \sqrt{2 \pi} c+c^{2}\right)^{-1} \varepsilon^{-2} / 4+\mathcal{O}\left(\varepsilon^{-1}\right)$ \\
$u_{4}$ & $-c^{-1} \varepsilon^{-2}+\mathcal{O}\left(\varepsilon^{-1}\right)$ & $(\sqrt{2 \pi}-c)\left(16 \pi+3 \sqrt{2 \pi} c+c^{2}\right)^{-1} \varepsilon^{-3}+\mathcal{O}\left(\varepsilon^{-2}\right)$ \\
\hline
\end{tabular}
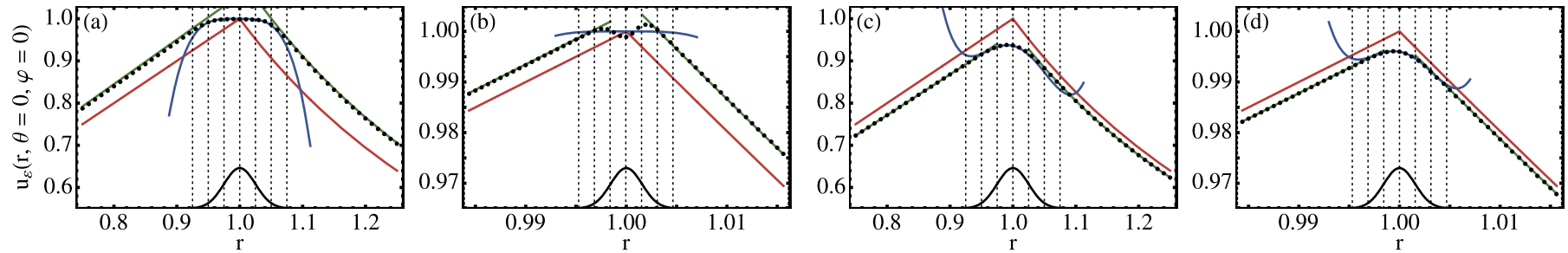

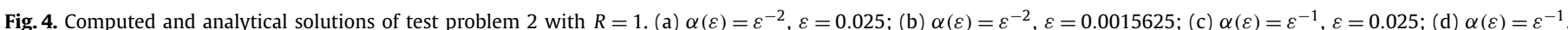

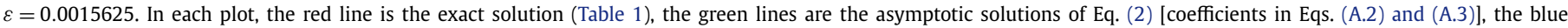

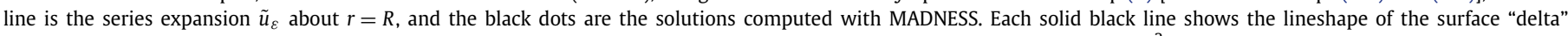

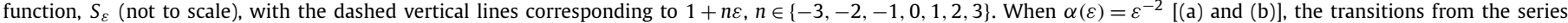

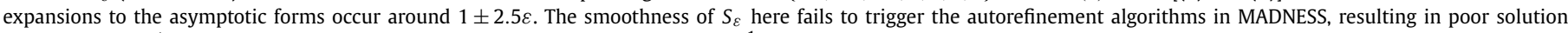

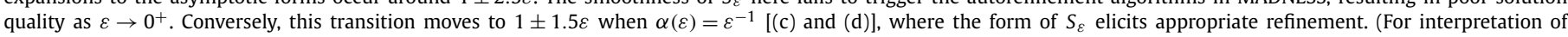
the references to color in this figure legend, the reader is referred to the web version of this article.)

Since all of the test problems have variable-separable analytical solutions, we approximate solutions of the DDA as series expansions about the surface $(r=R)$,

$\tilde{u}_{\varepsilon}(\mathbf{x})=\sum_{k} u_{k} f(\theta, \varphi)(r-R)^{k}$

Empirically, we find it necessary to include terms up to $k=4$. By construction, $\tilde{u}_{\varepsilon}$ will exhibit the correct asymptotic $r$-dependence; the question becomes the rate of convergence of the asymptotic coefficients to their correct values as $\varepsilon \rightarrow 0^{+}$. The three regions of interest are then (i) the interior, (ii) the exterior, and (iii) the boundary.

Focusing on problem 2 with $R=1$ (similar analyses can be performed for other problems and radii), the solution should behave as $u_{\varepsilon}(\mathbf{x}) \sim c_{0} r \cos (\theta)$ in the interior domain $(r \ll 1), u_{\varepsilon}(\mathbf{x}) \sim$ $c_{\infty} r^{-2} \cos (\theta)$ in the exterior domain $(r \gg 1)$, and as $u_{\varepsilon}(\mathbf{x})=\tilde{u}_{\varepsilon}(\mathbf{x})$ near the boundary $(r \approx 1)$. Calculating the series expansions, we find the asymptotic coefficients to be

$$
\begin{aligned}
c_{0}=\alpha(\varepsilon)\left(\frac{1-u_{0}}{3}-\frac{u_{2}}{3} \varepsilon^{2}-u_{4} \varepsilon^{4}+\mathcal{O}\left(\varepsilon^{6}\right)\right), \\
c_{\infty}=\alpha(\varepsilon)\left(\frac{1-u_{0}}{3}+\left(1-u_{0}-u_{1}-\frac{u_{2}}{3}\right) \varepsilon^{2}\right. \\
\left.-\left(u_{1}+3 u_{2}+3 u_{3}+u_{4}\right) \varepsilon^{4}+\mathcal{O}\left(\varepsilon^{6}\right)\right) .
\end{aligned}
$$

The expansion coefficients for $\alpha(\varepsilon)=c \varepsilon^{-2}$ are displayed in Table 2 . Substituting these expansion coefficients into Eqs. (A.2) and (A.3) shows that the asymptotic coefficients when $\alpha(\varepsilon)=c \varepsilon^{-2}$ correctly approach 1 as $\varepsilon \rightarrow 0^{+}$.

Figs. 4(a) and (b) display these analytical/asymptotic solutions, the exact solution, and the numerical solution obtained with MADNESS for $\varepsilon=0.025$ and $\varepsilon=0.0015625\left[\alpha(\varepsilon)=\varepsilon^{-2}\right]$, respectively. In both cases, the numerical solution is close to the exact solution at the boundary, but completely misses the derivative discontinuity, instead displaying non-physical smoothness. The solution quality thus degrades rapidly as we move away from the boundary, indicating an over-penalization at the boundary. This figure also shows that the series expansions transition to the asymptotic forms near $r=R \pm 2.5 \varepsilon$. The surface delta function $\left(S_{\varepsilon}\right)$ is quite smooth in these regions, and fails to invoke the autorefinement algorithms inside MADNESS. This is not problematic with large $\varepsilon$ [the numerical and analytical/asymptotic solutions agree, Fig. 4(a)]; however, MADNESS fails to adequately resolve the boundary layer as $\varepsilon$ becomes small, leading to poor solution quality [Fig. 4(b)].

This analytical analysis also exposes a more general problem (not specific to MADNESS) when $\alpha(\varepsilon) \propto \varepsilon^{-2}$. Moving to a distance $\mathcal{O}(\varepsilon)$ from the boundary, the series expansions show that $u_{\varepsilon}-d \sim$ $\mathcal{O}\left(\varepsilon^{2}\right)$. Multiplication by $\alpha(\varepsilon)$ makes the entire boundary term in the DDA appear as $\mathcal{O}\left(\varepsilon^{0}\right)$. The resulting loss of significant digits as $\varepsilon \rightarrow 0^{+}$also contributes to the unacceptable solution quality demonstrated in Fig. 4(b). We note that numerical calculations in MAPLE are able to reproduce the analytical/asymptotic solution in Fig. 4(b), but only with highly-increased precision.

Owing to the over-penalization and undesirable cancellation when $\alpha(\varepsilon) \propto \varepsilon^{-2}$, we now consider penalties of the form $\alpha(\varepsilon)=$ $c \varepsilon^{-1}$. The series expansion coefficients are given in Table 2; the asymptotic coefficients again approach unity as $\varepsilon \rightarrow 0^{+}$. Note that this reduced penalty also alleviates the cancellation problems experienced when $\alpha(\varepsilon) \propto \varepsilon^{-2}$.

Figs. 4(c) and (d) display similar information as panels (a) and (b), respectively, for $\alpha(\varepsilon)=\varepsilon^{-1}$. First, we see larger errors at the boundary, indicating reduced penalization there, as well as a more reasonable approximation of the derivative discontinuity. Second, the asymptotic solutions are closer to the exact solution, translating to better solution quality in the interior and exterior domains. Finally, the transitions from series expansions to asymptotic forms now occur near $r=R \pm 1.5 \varepsilon$, where $S_{\varepsilon}$ triggers MADNESS's autorefinement algorithms. With adequate resolution of the boundary layer, penalties of the form $\alpha(\varepsilon) \propto \varepsilon^{-1}$ produce acceptable solutions in MADNESS, even as $\varepsilon$ becomes small [Fig. 4(d)].

\section{References}

[1] X. Li, J. Lowengrub, A. Rätz, A. Voigt, Commun. Math. Sci. 7 (2009) 81.

[2] http://code.google.com/p/m-a-d-n-e-s-s/.

[3] R.J. Harrison, G.I. Fann, T. Yanai, Z. Gan, G. Beylkin, J. Chem. Phys. 121 (2004) 11587.

[4] R.J. Harrison, G.I. Fann, T. Yanai, G. Beylkin, Multiresolution quantum chemistry in multiwavelet bases, in: Lecture Notes in Computer Science, vol. 2660, Springer-Verlag, Heidelberg, 2003, pp. 103-110. 
[5] T. Yanai, G.I. Fann, Z. Gan, R.J. Harrison, G. Beylkin, J. Chem. Phys. 121 (2004) 6680.

[6] G.I. Fann, et al., J. Phys.: Conf. Ser. 180 (2009) 012080.

[7] J. Jia, J.C. Hill, G.I. Fann, R.J. Harrison, Multiresolution fast methods for a periodic 3-d Navier-Stokes solver, in: Q. Guo, Y. Guo (Eds.), DCABES 2009: The 8th International Symposium on Distributed Computing and Applications to Business, Engineering, and Science, 2009, pp. 13-16.

[8] A. Rätz, A. Voigt, Commun. Math. Sci. 4 (2006) 575.

[9] B. Alpert, G. Beylkin, D. Gines, L. Vozovoi, J. Comput. Phys. 182 (2002) 149.

[10] Y. Saad, M.H. Schultz, SIAM J. Sci. Stat. Comput. 7 (1986) 856.
[11] J.D. Jackson, Classical Electrodynamics, 3rd edition, John Wiley \& Sons, 1999, Chapter 2.1.

[12] S. Grafström, J. Appl. Phys. 91 (2002) 1717.

[13] M. Sukharev, T. Seideman, J. Phys. Chem. A 113 (2009) 7508.

[14] M.W. Schmidt, et al., J. Comput. Chem. 14 (1993) 1347.

[15] B. Pettinger, B. Ren, G. Picardi, R. Schuster, G. Ertl, Phys. Rev. Lett. 92 (2004) 096101.

[16] M.G. Reuter, M. Sukharev, T. Seideman, Phys. Rev. Lett. 101 (2008) 208303.

[17] J.M. Montgomery, T.-W. Lee, S.K. Gray, J. Phys.: Condens. Matter 20 (2008) 323201. 